\title{
The need for oral and dental hygiene in patients with ischemic cardiomyopathy - preliminary study
}

\author{
Ioana Voinescu', Dumitru Ferechide', Irina Adriana Beuran', Mihai Burlibasa', \\ Simion Gheorghe Dumitru' ${ }^{2}$, Radu Costea ${ }^{1}$, Iuliana Babiuc ${ }^{1}$, Oana Eftene $^{1}$, \\ Viorel Stefan Perieanu', Irina Donciu', Madalina Violeta Perieanu', Oana-Cella Andrei', \\ Ruxandra Stanescu' ${ }^{1}$ Narcis Marcov ${ }^{1}$, Elena-Cristina Marcov ${ }^{1}$, Olivia Popoviciu' ${ }^{1}$ \\ 1"Carol Davila" University of Medicine and Pharmacy, Bucharest, Romania \\ ${ }^{2}$ Dental practice, Brașov, Romania
}

\begin{abstract}
Introduction. Most patients over 40 years old who present for specific treatments in the dental offices have at least one general condition. Specialized research has shown that atherosclerosis is more common in patients with periodontal disease, which suggests that periodontal and cardiovascular disease may have similar causes. Purpose. Given the close connection between the two conditions, we have tried to draw the attention of patients to the importance of the prophylactic maneuvers performed by him or by specialized personnel and we also want to emphasize the importance of the dental hygienist in this process.

Material and method. In order to carry out this study, a questionnaire with predetermined answers was used, applied to 57 patients with ischemic cardiomyopathy and who presented for specialized treatments in dental offices.

Results and discussions. The answers really reflect how the patients understood the connection between the two conditions and how they perform the oral and dental hygiene.

Conclusions. Given that many of the respondents do not perform the hygiene procedures correctly or incompletely, there is a need for a medical staff to discuss with patients to educate them on the importance and the way of hygiene and to perform the oral and dental hygiene procedures, in short the dental hygienist.
\end{abstract}

Keywords: ischemic cardiomyopathy, periodontal disease, dental hygienist

\section{INTRODUCTION}

Nowadays, there are fewer and fewer cases where patients who go to a dental office for a specialized treatment enjoy a perfect state of health. Specifically, most of the patients we are dealing with in daily dental practice accuses at least one general condition, a situation that is common in patients over 40 years old (1-5). Thus, the recognition of the clinical symptomatology that characterizes the systemic diseases of the patients, supposes from the dentist a complex medical knowledge, his capacity to correlate his knowledge with the specialized treatment that he will apply to the patients, with the patient's perception of the disease they are suffering from, the medication they are taking, as well as the side effects that this medication can cause (1-6).

Although the dentist does not have to establish the diagnosis of the general disease, it is his obligation to send the patient to the general practitioner, when he suspects an undiagnosed general condition or when the known one of the patient may be a risk factor in the specialized therapy, which is to be performed in the dental office (1-6). We must also take into account the mental stress of the patient, stress caused by contact with the dental office, with the 
environment and its specific treatments, stress that can cause the patient a variety of problems and complications with varying severity.

\section{GENERAL DATA}

It is known that there is a connection between dental and periodontal diseases and some systemic diseases: since the time of Hippocrates it was known that dental pathology, especially the pyorrhea, has repercussions on the health of other organs of the human body, mainly in the joints (1-6).

By studying the data from the specialized literature, it has been established very precisely that everything that means periodontal disease can have adverse effects, some with remote manifestations, but with systemic consequences of lesser or greater extent. Thus, periodontal disease can be characterized as a bacterial infection with oral and dental localization, affecting a very large segment of the population aged between 20 and 80 years, being one of the main causes which, in the absence of local and / or general treatment, it can ultimately lead to tooth loss. $(1-5,7,8)$.

However, it is known that between periodontal diseases and systemic conditions there is a close correlation: periodontal diseases can aggravate systemic disorders (cardiovascular diseases, diabetes), just as systemic diseases (osteoporosis etc.) can influence periodontal disease (1-6). Particular importance is given in this context to atherosclerosis, an evolutionary disease that affects large and medium-sized vessels, which can cause ischemic brain, cardiac or extremity injury or thrombosis and heart attack so that it can eventually cause death (1-6).

Thus, the researches carried out in this field has shown that atherosclerosis is more common in patients with periodontal disease, which suggests that periodontal and cardiovascular disease may have similar causes $(1-3,9,10-11)$.

\section{PURPOSE}

Several biological mechanisms are described in the literature, which may explain the link between cardiovascular disease and periodontal disease (13,9-13).

1. Systemic factors. Atherosclerosis is an inflammatory condition. During the inflammatory process, monocytes migrate to the underlying tissues, where smooth muscle cell proliferation occurs. The consequences of the migration of inflammatory cells into the circulation of large vessels are represented by fatty vascular degeneration, but also by intravascular coagulation.
2. The role of infectious agents. Existing bacteria in the periodontal tissue may enter the systemic circulation, can cause direct injury to the vascular endothelium, and may partially activate the inflammatory immune response associated with atherosclerotic disease. It was found that $45 \%$ of atheromatous plaques contain at least one of the bacteria involved in periodontal disease (Porphyromonas gingivalis, Prevotella intermedia, Bacteroides forsytus, Actinobacillus actynomicetemcomitans), and $72 \%$ contain bacterial DNA from one of these microorganisms. In general, these atheromatous plaques with the bacteria existing at this level are incriminated in the production of ischemic cardiomyopathy (1-3,14-17).

Thus, ischemic cardiomyopathy represents myocardial distress caused by narrowing (stenosis) and / or blockage (occlusion) of the coronary arteries. The main cause of coronary artery disease is atherosclerosis and consists, as we have discussed before, in the deposition of cholesterol on the inner wall of the arteries which, in combination with certain blood cells and the blood vessel wall, form the atheromatous plaque. It narrows and eventually blocks the artery, impeding blood flow to the myocardium (18).

But, the specialized dental treatment for patients with ischemic cardiomyopathy, including the dental-alveolar surgery treatments, must follow a classic protocol, which we find in the specialized guide published by the Romanian College of Dentists (19). This very complete and particularly laborious guide, elaborated by a highly prestigious Romanian specialist group, is the basis of all the specialized dental treatments, regardless of the condition the patient is suffering from.

Given the close connection between periodontal diseases (more precisely, the microbial germs involved in the periodontal disease pathology), atheromatous plaques and ischemic cardiomyopathy, we tried to guide patients with such medical problems, who presented for specialized treatments in dental private clinics where we, the authors, carry out our activity, on some prophylactic maneuvers, of compulsory oral and dental hygiene, which must be performed by them both at home, daily, but also professionally, at certain time intervals, in the dental offices by specialized personnel. In fact, this is also the objective of our study.

\section{MATERIAL AND METHOD}

\section{Preliminary considerations}

Generally speaking, prophylaxis defines the prevention of a disease, and dental prophylaxis is a 
method of preventing dental diseases, including periodontal diseases. The objective of dental prophylaxis is to identify and remove harmful substances from both above and below gingival margin. These harmful substances are represented by the microbial dental plaque, the dental calculus, food residues, substances that, both due to their very irritating character and an absolute microbial load (see the microbial dental plaque and the dental calculus), are the main causes of the beginning and the maintenance of periodontal disease (20). There is a close connection between the microbial germs involved in the etiology of periodontal disease, atheromatous plaques and ischemic cardiomyopathy.

Dental prophylaxis for patients suffering from ischemic cardiomyopathy is performed every 4-6 months in 2 ways:

- At home, through the patient's personal efforts, through proper brushing techniques using either the classic or the electric toothbrush, but also complementary methods as dental floss, mouthwash, gum stimulators, water flosser, etc.

- In dental offices, by the specialized team (dentist, oral and dental prophylaxis nurse), using the specific instrumentation: ultrasonic scaler when the patient's health allows it; manual and rotary instrumentation.

Regarding the oral prophylaxis performed through the patient's personal efforts, it should be noted that both dental practitioners and especially dental nurses and oral and dental prophylaxis nurses are the ones who provide patients with oral hygiene instructions, and they have to master various methods, which patients can use daily, to clean their teeth. During the training phase, the proper brushing technique will be established for each patient, whether it's using conventional toothbrushes or electric toothbrushes. At the same time, the use of the microbial dental plaque disclosing substances should not be omitted. Patients suffering from ischemic cardiomyopathy, who perform their brushing at home alone, can be divided into 4 categories:

1. Patients who perform the correct dental brushing and who thoroughly remove the dental microbial plaque, without injuring their soft tissues;

2. Patients who remove their dental plaque relatively well and conscientiously, but there are still neglected areas;

3. Very dynamic, aggressive patients, who remove the dental plaque efficiently, but with repeated and, sometimes extremely stressed, soft tissue damage;
4. Slow patients, not interested in oral hygiene, who do not brush their teeth regularly or do not follow the correct dental brushing techniques.

Also for a good maintenance of oral hygiene at home for patients suffering from ischemic cardiomyopathy, besides the classic and electric toothbrushes, besides toothpaste, it is also recommended a number of complementary methods, such as: dental floss with or without support; buccal mirrors with which the patient can evaluate the efficiency of dental brushing; straight or conical interdental brushes; gingival stimulators to stimulate gum blood circulation; water flosser for debris removal with water jet and for distribution of drug substances in spaces inaccessible to classic and electric toothbrushes; mouthwashes comprising various antimicrobial agents.

Regarding the professional dental prophylaxis performed in the dental office by the specialized team (dentist, oral and dental prophylaxis nurse) for patients with ischemic cardiomyopathy, it consists of dental scaling and brushing procedures and must be started with a rigorous control of the microbial dental plaque index, by using dental plaque disclosing substances. Then, depending on the condition of the patient, we proceed to the actual scaling operations (to the proper oral and dental cleaning). If the patient has a pacemaker, the ultrasonic scaling will not be used, as it may interfere with the pacemaker's functioning. At the same time, if the patients are on an anticoagulant medication that has not been interrupted, cleaning procedures with manual and rotary instruments must be very gentle, in order to avoid damaging soft tissues, and thus bleeding. Depending on the complications arising from ischemic cardiomyopathy, the dentist and / or the nurse for oral and dental prophylaxis, using the guide of good practices in dental medicine that we discussed above, must be careful how and if they use anesthetic substances to perform the scaling operations. We will not insist on the professional scaling tools (manual, rotary, ultrasonic) used in the dental offices, because it is well known by all the dental practitioners.

Instead, it is necessary to discuss the necessity of the dental hygienist profession existence in Romania, as this profession has no correspondence at this moment in our country. Even though a large part of the dentists do not agree with the existence of dental hygienists in Romania pretending that they could take over a large part of their tasks, and therefore of their income. In the European Union there are 19 countries that recognize this profession. 
Studying the specialized literature, according to some authors, the most important role of the dental hygienist is to perform the non-surgical periodontal therapy, which includes several procedures, following the periodontal clinical examination: dental scaling and root planing, cleaning of the periodontal pockets in the patients with periodontal diseases, professional brushing. By all these procedures, the presence of the dental microbial plaque is significantly reduced, the dental calculus is removed, the periodontal disease is prevented or, if it is already present, clinical manifestations are diminished and complications that may occur, including loss of teeth, are prevented (21).

Other tasks of the dental hygienist would be the following (21):

- preventive services, which promote hygiene methods and the importance of maintaining oral health;

- educational services, through which the dental hygienist will be the one who will educate his patients on the prevention of dental problems, by implementing correct brushing techniques, associated with the complementary methods (dental floss, mouthwash, water flosser, etc.) and which will teach them how to clean their teeth, in the case of patients with fixed orthodontic appliances, with fixed prosthetic restorations, or teach patients how to clean their mobile prosthetic restorations (total and/or partial dentures);

- therapeutic services: these consist of various procedures, by which certain conditions that can affect the health of the periodontal tissues are treated;

- teamwork: a dental hygienist will collaborate with the dentist, orthodontics specialist, with the specialist in periodontology, for an interdisciplinary approach to clinical cases, in order to provide patients with the best oral health care.

\section{Questionnaire and subjects data}

The study is based on a rigorous questionnaire based on 7 closed-ended questions. The study group consists of 57 patients known to be suffering from ischemic cardiomyopathy, who presented for specialized treatments in the dental offices where, we, the authors, carry out our activity. Subjects ranged in age from 45 to 76 years. Of these, 37 subjects (representing 64.91\%) were male, while the remaining 20 subjects (representing 35.09\%) were female (Fig. 1). The study was conducted over a period of 2 years, between January 2017 and De- cember 2018, it was conducted subject to anonymity and benefited from the informed consent of the subjects involved in the study.

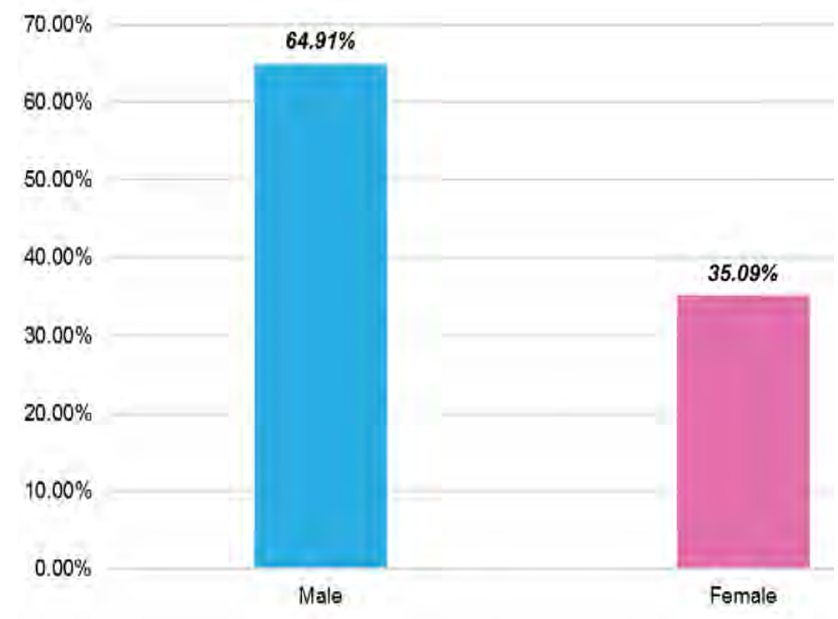

FIGURE 1. Gender distribution of the subjects included in the study

The questionnaire applied to the 57 subjects was as follows:

1. How did it was explained by the dental medical team to you the connection between certain microorganisms that we find in the etiology of gingival disorders, atheroma plaques and ischemic cardiomyopathy? a. These microorganisms are found in atheroma plaques deposited on the pathway of coronary vessels and are also involved in the etiology of ischemic cardiomyopathy. b. They are harmless bacteria and we find them only at the periodontal level. c. We find these microorganisms in atheroma plaques deposited on the path of coronary vessels that produce ischemic cardiomyopathy, but are extremely aggressive only at the periodontal level.

\section{Correct answer: $a$.}

2. What do you think the goal of dental prophylaxis is? a. Removal from both above and below gingival margin of dental microbial plaque. b. Removal of dental calculus. c. Removal of high dental fillings. $\boldsymbol{d}$. Removal of fixed prosthetic restorations with mucosal contact; $\boldsymbol{e}$. Removal of food debris.

Correct answers: $a, b, e$.

3. How have you been told that oral prophylaxis should be performed for you (patients suffering from ischemic cardiomyopathy)? a. At home, through the patient's personal efforts; $\boldsymbol{b}$. In the dental offices, by the specialized team (dentist, oral and dental prophylaxis nurse); $c$. Both in the dental and general practice offices. $\boldsymbol{d}$. Periodically in the dental office, every 4-6 months.

Correct answers: $a, b, d$. 
4. After studying the information presented, in which category of patients you find yourself? a. Patients who perform the correct dental brushing and who thoroughly remove the dental microbial plaque, without injuring their soft tissues; $\boldsymbol{b}$. Patients who remove their dental plaque relatively well and conscientiously, but there are still neglected areas; $c$. Very dynamic, aggressive patients who remove the dental plaque efficiently, but repeated and sometimes very pronounced soft tissue injury; d. Slow patients, uninterested in oral hygiene who do not brush their teeth regularly or do not follow the correct dental brushing techniques.

5. To maintain good oral hygiene at home for you (patients suffering from ischemic heart disease), you should use: a. classic and/or electric toothbrushes; b. rotary instruments; c. Ultrasonic instruments; $\boldsymbol{d}$. Complementary methods, such as dental floss, interdental brushes, water flosser.

\section{Correct answers: $\boldsymbol{a}, \boldsymbol{d}$.}

6. Professional dental prophylaxis for patients suffering from ischemic cardiomyopathy performed in the dental office by the specialized team (dentist, oral and dental prophylaxis nurse) includes the following true or false aspects: a. It starts with a very strict control of the of dental microbial plaque index, by using the plaque disclosing substances; $\boldsymbol{b}$. It is performed only with ultrasonic instruments, for patients with pacemakers; $c$. If the patients are on an anticoagulant medication and this has not been interrupted, the manual and rotary scaling and hygiene operations must be very calm, gentle, in order to avoid damaging the soft tissues, and thus bleeding.

\section{Correct answers: $a, c$.}

7. In your vision, what do you think the role of a dental hygienist should be? a. To ensure preventive and educational services; $\boldsymbol{b}$. To ensure various small-scale therapeutic maneuvers, such as scaling and professional brushing; $c$. To be part of the interdisciplinary team, together with the dentist, the orthodontics specialist, with the specialist in periodontology; d. To deal with the financial problems of the dental office?

Correct answers: $a . b, c$.

\section{RESULTS AND DISCUSSIONS}

Following the evaluation of the answers from the 7 questions, some aspects have resulted.

It is noteworthy that over half of the patients included in the study (38 - representing 66.67\%) were attentive and understood the explanations given by the dental medical team regarding the connection between the microorganisms involved in the etiology of the gingival diseases and the atheroma plaques, respectively the ischemic cardiomyopathy. One third of patients (19 - representing 33.33\%) understood that the incriminated microorganisms are extremely aggressive only at the periodontal level (Fig. 2).

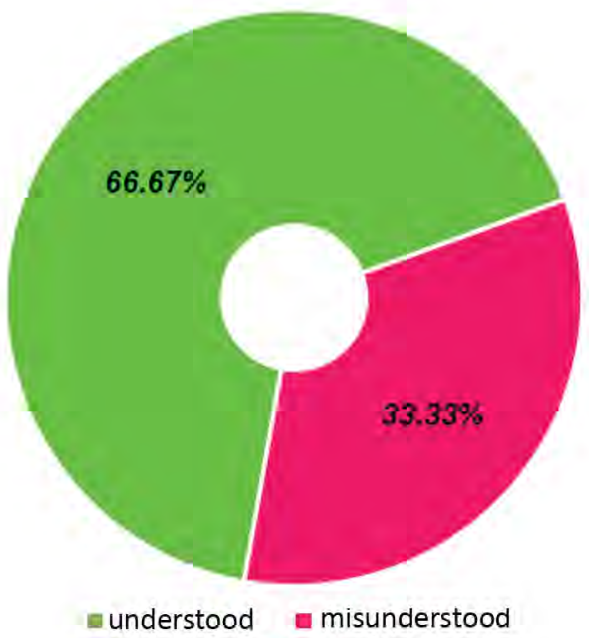

FIGURE 2. Understanding the connection between microorganisms in gingival diseases and atheroma plaque, respectively ischemic cardiomyopathy

Regarding the goals of dental prophylaxis, all patients responded correctly, namely: removal of the microbial plaque, removal of calculus and food debris.

To the third question related to oral and dental prophylaxis in patients with ischemic cardiomyopathy, the majority of patients (50 - representing $87.72 \%$ ) answered correctly: by the patient, by the team of dental specialists and every 4-6 months. Only 7 patients (representing 12.28\%) considered that prophylaxis should be performed by the general practitioner and the dentist at 4-6 months (Fig. 3).

The self-evaluation performed by the patients based on the information presented by the medical team led to the following results:

- 12 patients (representing 21.05\%) perform the correct dental brushing without damaging the soft tissues;

- 20 patients (representing 35.09\%) remove the dental plaque relatively well but there are also neglected areas;

- 10 patients (representing 17.54\%) remove the dental plaque efficiently with the accentuated and even repeated damage of the soft tissues;

- 15 patients (representing 26.31\%) not interested in oral hygiene (Fig. 4). 

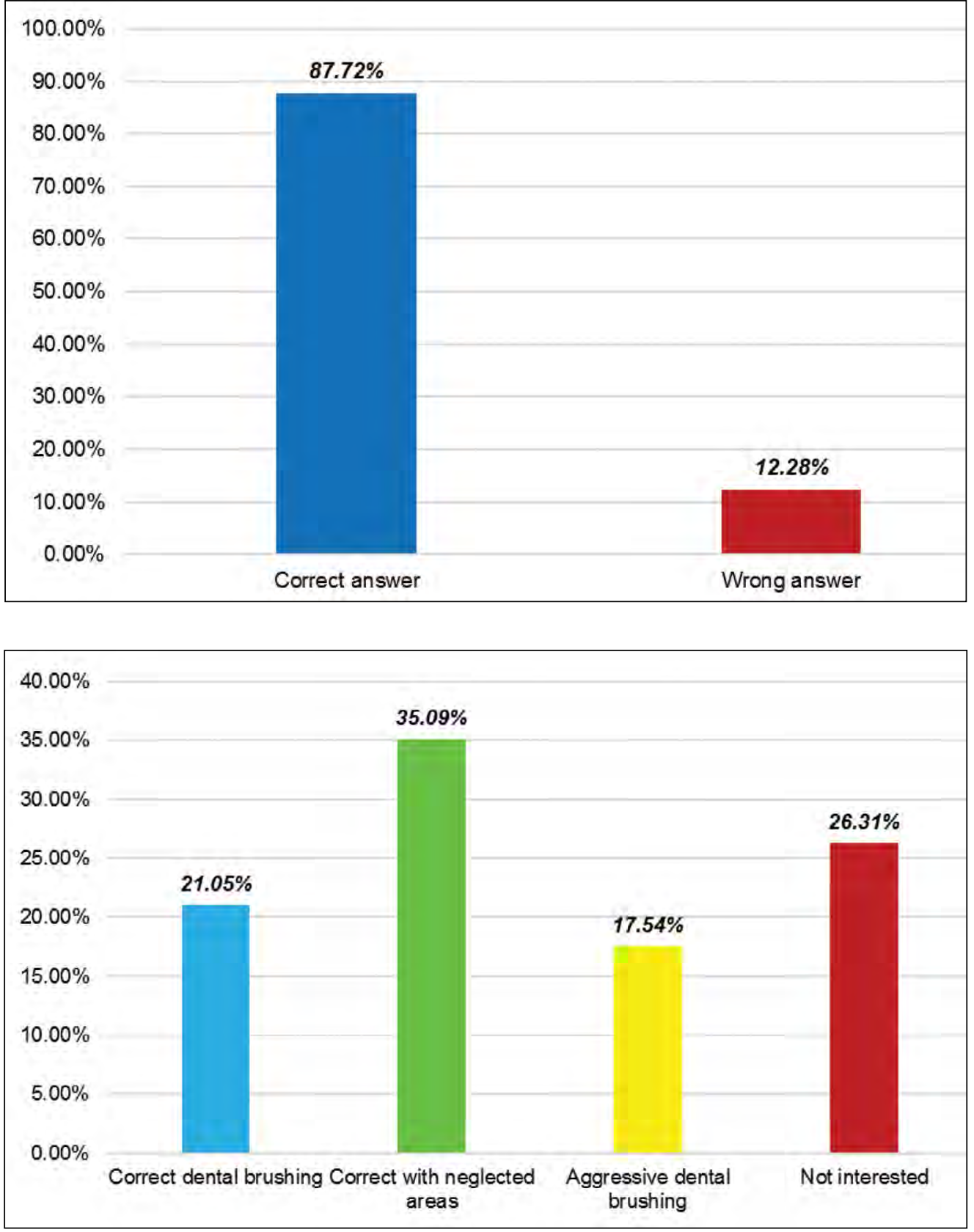

FIGURE 3. Oral and dental prophylaxis in patients with ischemic cardiomyopathy
FIGURE 4. Self-assessment by patients of the degree of hygiene they perform from the perspective of removing the dental plaque and protecting the soft tissues
Another question that all respondents answered correctly is that of maintaining oral hygiene at home (toothbrushes and complementary methods).

With regard to professional dental prophylaxis in patients with ischemic cardiomyopathy, most patients (48 - representing 84.21\%) responded correctly, namely: strict initial control followed by gentle hygiene procedures to avoid soft tissue damage and thus bleeding. Only 9 patients (representing $15.79 \%$ ) answered incorrect, probably from the partial understanding of the explanations given by the medical team (Fig. 5).

To the last question related to the role or roles that the dental hygienist should play, all the respondents to the study offered the correct answers.

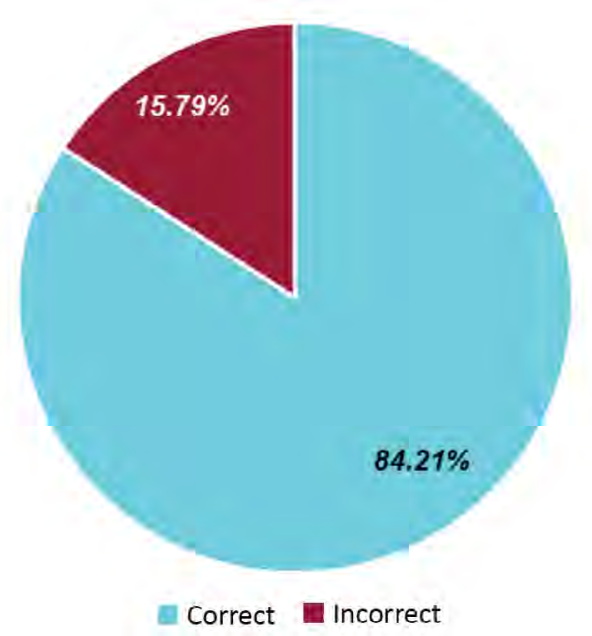

FIGURE 5. Understanding the term of professional dental prophylaxis 
Analyzing the answers offered by the 57 participants in the study we were able to conclude some aspects. The sex distribution of patients suffering from ischemic cardiomyopathy was as follows: 37 subjects (representing 64.91\%) were male, while the remaining 20 subjects (representing 35.09\%) were female, these percentages are consistent with the data in the specialized literature.

Most of the patients involved in the study $(66 \%$ representing 2/3) agreed that the microbial side plays an important role in the occurrence of ischemic cardiomyopathy, understanding the meaning of the oral prophylaxis in this case, while the rest of $33.33 \%$ accepted the implementation of the oral and dental prophylaxis maneuvers, only at the recommendation of the dental medical team, without taking into account or without understanding the argument presented by them.

Over $85 \%$ of the patients involved in the study understood all the information regarding the oral prophylaxis: this is done personally, daily at home based on the information provided by the dental medical team (dentist, dental nurse, oral and dental prophylaxis nurse) and every 4-6 months in the dental office, by the specialized team.

Although the majority of patients (73.68\%) achieve a good removal of the bacterial plaque, there are differences in the degree of correctness regarding self-hygiene.

Most of the patients involved in the study $(35.09 \%)$, have neglected areas, a slightly smaller group $(21.05 \%)$ correctly removes the bacterial plaque without damaging the soft tissues and $17.54 \%$ perform an aggressive brushing, effective but which results in the damage of soft tissues. Only a quarter of respondents are not interested in oral hygiene or how they perform it.

Considering that, the professional oral and dental hygiene measures performed in the dental office by the specialized team (dentist, oral and dental prophylaxis nurse) present a series of interdictions for patients suffering from ischemic cardiomyopathy (avoiding the use of the ultrasonic instrument for patients with pacemakers, as well as avoiding more aggressive manual hygiene maneuvers for patients with anticoagulant medication and who have not interrupted it), it is more recommended to check the dental plaque index every 4-6 months, with light and gentle removal maneuvers of the dental microbial plaque (soft deposits) and of the dental calculus with the help of manual and rotary instru- ments, to prevent the injury of the soft tissues and bleeding.

The basic in maintaining a good oral and dental hygiene, is the prophylaxis made at home, performed by patients suffering from ischemic cardiomyopathy, based on the guidance provided by the specialized team (dentist, oral and dental prophylaxis nurse): dental brushing with the help of the classic and electric toothbrushes, the use of the classical or on support dental floss, the interdental brushes, the gum stimulators, the water flosser, the mouthwashes, etc.

The training should be intensified, regarding the oral and dental prophylaxis at home, by implementing very effective programs, for the correct application of the brushing techniques for all categories of patients, including for those suffering from ischemic cardiomyopathy: starting in kindergartens and schools and ending with the use of brochures, books and/or media, to send a message of such magnitude and importance.

Reinsertion as soon as possible in the Universities of Medicine and Pharmacy from Romania of the university programs for oral and dental prophylaxis assistance, which have completely disappeared from our country during the last 2 years, due to an acute lack of interest from the decision factors.

Creating reconversion programs for general nurses in dental nurses or oral and dental prevention nurses is a necessity.

The introduction of "dental hygienist" profession in the COR (Classification of Occupations in Romania) which, at this moment, has no equivalent in Romania. We, the authors of this material, believe that the introduction of the dental hygienist profession in Romania is a high priority.

\section{CONCLUSIONS}

Given that many of the respondents do not perform the hygiene procedures correctly or incompletely, there is a need for a medical staff to discuss with patients to educate them on the importance and the way of hygiene and to perform the oral and dental hygiene procedures, in short the dental hygienist.

\section{Acknowledgement}

In this article, all the authors have equal contribution with the first author. 


\section{REFERENCES}

1. Burlibaşa M. Corelaţia dintre diferitele afecțiuni generale și managementul dentar al pacientului. În: Managementul dentar al pacientului cu probleme medicale complexe. Coordonator: Bodnar D.C., p. 39-52, 102, Editura Ars Docendi, Bucureşti, 2012.

2. Burlibașa $M$, Bodnar DC. Aspecte teoretice și practice privind corelația dintre diferitele afecțiuni generale și managementul general al pacientului. În: Progrese în medicina dentară, vol. VI. Coordonatori. Dumitrache A.M., Burlibașa M., Sfeatcu R.I., Bodnar T., p. 45-54, Editura Ars Docendi, București, 2012.

3. Sfeatcu R, Burlibaşa M, Tofan $C$ et al. Calitatea vieții, un concept multidisciplinar. Sibiul Medical. 2007; Vol. 18, No. 1, p. 18-20.

4. Anghel M. Diagnosticul oral, p. 159-195. Editura Orizonturi Universitare, Timișoara, 2004.

5. Little WJ, Falace AD, Miller SC et al. Dental management of the medically compromised patient. $7^{\text {th }}$ Ed. St. Louis, Mosby/Elsevier, 2008.

6. Dumitriu HT. Parodontologie, Ed. Viaţa Medicală Românească, Bucureşti, 1999.

7. Holtfreter B, Albandar JM, Dietrich T et al. Standards for reporting chronic periodontitis prevalence and severity in epidemiologic studies - Proposed standards from the Joint EU/USA Periodontal Epidemiology Working Group. J Clin Periodontol 2015; 42(5): 407-412.

8. Dye BA, Selwitz RH. The relationship between selected measures of periodontal status and demographic and behavioural risk factors. J Clin Periodontol 2005; 32(7): 798-808.

9. Söder PÖ, Söder B, Nowak J, Jogestrand T. Early carotid atherosclerosis in subjects with periodontal disease. Stroke. 2005; 36(6): 1195-1200.

10. Bradley FB, Amy LD, David JV. Review: High-risk periodontal pathogens contribute to the pathogenesis of atherosclerosis. Postgraduate Medical Journal 2017; 93: 215-220.
11. Lăcustă V, Lupan I, Fala V. Paradigma medicinei integrative în parodontologia modernă. Editura Akademos, 2015; 2(37): 94-98.

12. Williams RC, Mahan CJ. Periodontal disease and diabets in young adults. J Am Med Assoc. 1960; 20(172): 776-778.

13. Offenbacher $\mathrm{S}$, Beck JD, Moss $\mathrm{K}$ et al. Results from the periodontitis and vascular events (PAVE) Study: A pilot multicentered, randomized, controlled trial to study effects of periodontal therapy in a secondary prevention model of cardiovascular disease. J Periodontol 2009; 80(2):190-201.

14. Jaume C, Guillermo R, Santiago A, Joan C. Ischemic Cardiomyopathy: A Clinical Nuclear Cardiology Perspective. Rev Esp Cardiol 2009; 8(62): 903-917.

15. Fives-Taylor P, Meyer D, Mintz K. Characteristics of Actinobacillus actinomycetemcomitans invasion of and adhesion to cultured epithelial cells. Adv Dent Res 1995; 9(1): 55-62.

16. Ebersole JL. Humoral immune responses in gingival crevice fluid: local and systemic implications. Periodontol 2000 2003; 31:135-166.

17. Haraszthy VI, Zambon JJ, Trevisan M et al. Identification of periodontal pathogens in atheromatous plaques. J Periodontol 2000; 71(10):1554-1560.

18. Cardioclinic. Cardiopatia ischemică. Cardioclinic. [Interactiv] [Citat: 12 02 2019.] https://www.cardioclinic.ro/boli/cardiopatia-ischemica/.

19. CMDR. Ghiduri de practică. CMDR. [Interactiv] [Citat: 1202 2019.] https://cmdr.ro/documente/chirurgie-orala-dento-alveolara/.

20. Miyasaki-Ching CM. Elemente clinice de stomatologie. Editura All Educational, București, 2001.

21. Doctorul de dinţi, igienist dentar. [Interactiv] [Citat: 1202 2019.] https:// www.doctoruldedinti.info/igienistul-dentar-si-de-ce-ar-trebui-sa-ilintalnim-mai-des/. 